\title{
Sexual behavior: postcopulatory heart rate in the male and female rat'
}

\author{
D. Thomas Law and James E. Skinner \\ CLAREMONT GRADUATE SCHOOL
}

\begin{abstract}
Postcopulatory heart rates were recorded from male and female rats. The peak rate was characteristically distinctive for abortive mounts, intromissions, and ejaculations, as was the temporal pattern. The first and last intromissions in the copulatory sequence were not significantly different but heart rate following ejaculation was. The patterns and peak rates differed for the two sexes.
\end{abstract}

\section{Problem}

The stereotyped copulatory sequence in the rat has been intensively studied by both Larsson (1956) and Beach (1959). Typically, the male rat mounts, thrusts briefly with the pelvis, and dismounts, repeating this performance several times before the sequence is finally terminated by ejaculation. Usually, especially in experienced males, mounting is accompanied by insertion of the penis in the vagina (intromission), but this is not always the case. After a refractory period of several minutes (the post-ejaculatory interval), a second sequence is usually initiated by the male. Successive copulatory sequences follow until the male is sexually exhausted. Larsson and Beach have independently proposed that successive intromissions in the sequence result in successive increments in a central excitatory mechanism which accumulate until a critical level is reached and ejaculation results.

In support of the hypothesis, Larsson (1956) observed that the intervals between successive intromissions become shorter as the time of ejaculation is approached, reflecting increasing levels in the central excitatory mechanism. Beach \& Whalen (1959) inter rupted the normal sequence for various intervals of time; thereby increasing the number of intromissions to ejaculation and presumably decreasing the central excitatory levels.

Previous work in other animals (Ford, 1952; Larsson, 1956; Mendelsohn, 1896) established a reliable relationship between the heart rate and both subjective and objective measures of sexual arousal and excitement. It was the purpose of the present study to verify this relationship in the rat and to study the hypothesized increments in sexual central excitatory states through parallel increments in heart rate.

\section{Method}

Sixteen male and 16 female hooded rats were allowed to mate while continuous heart rate recordings were taken. The recording wires were soldered onto wound clips which were clipped into the sides of both rats prior to testing. The wires apparently did not hinder performance.
Previously ovariectomized females were brought into receptive states with subcutaneous diethyl stilbestrol (500 micrograms) followed in $48 \mathrm{hr}$. by progesterone (1.0 mg).

\section{Results}

Figure 1 shows the average male heart rate for each second during the 20-sec. interval following termination of the coital contact for each of the following coital events: first mount with intromission in the sequence (I1), last mount with intromission before ejaculation $\left(\mathrm{I}_{\mathrm{E}-1}\right)$, mount without intromission $(\mathrm{M})$, and mount with ejaculation (E). Figure 2 shows similar data for females.

Heart rate rises abruptly for both sexes immediately after all classes of copulatory activity. For males, the peak rate occurs somewhat later $(4-5 \mathrm{sec}$.) and persists longer (6-7 sec.) than for females $(2 \mathrm{sec}$. and 4 sec. respectively). The trends of change in the male are generally smoother. Peak rates and resting rates (about 510) are higher than the female's (about 460), but the latter's range is greater. Male heart rates range some 60 beats $/ \mathrm{min}$. from peak to resting, while the female varies about 80 beats $/ \mathrm{min}$.

During the intercopulatory interval (ICI) following copulation the heart rate curves describe functions characteristic of the preceding sexual activity: unsuccessful mounts are followed by low initial rates, low peak rates, and rapid return to a low resting rate. A secondary increase in rate may be seen late in the interval; this

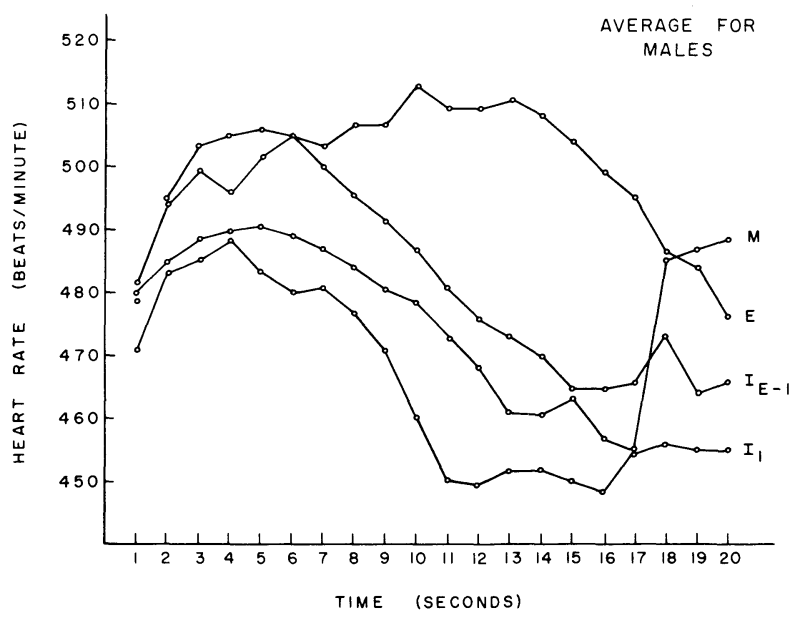

Fig. 1. Post-coital heart rate for males. $M=$ mounts without intromission; $I_{1}=$ initial intromission of copulatory series; $\mathrm{I}_{\mathrm{E}-1}=$ penultimate intromission of series; $\mathrm{E}=$ ejaculation. 


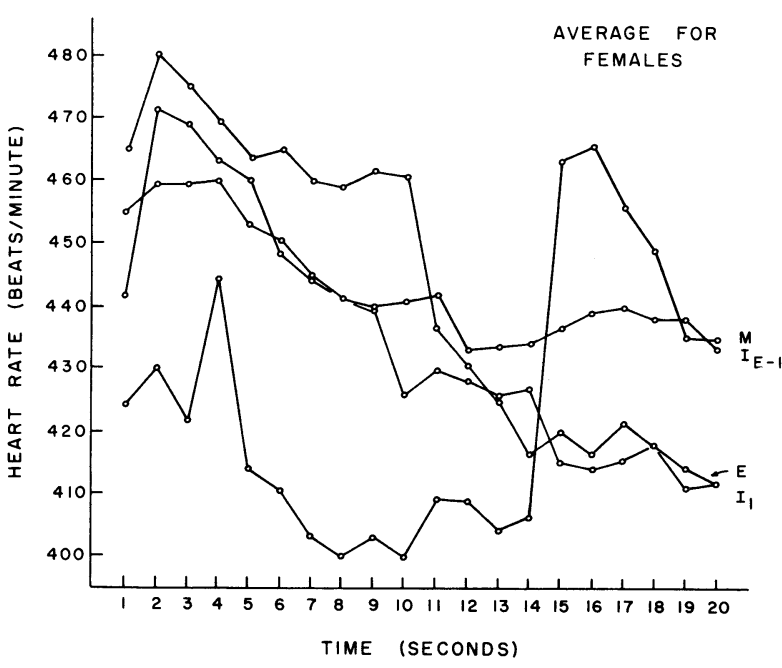

Fig. 2. Post-coital heart rate for females. $M=$ mounts without intromission; $\mathrm{I}_{1}=$ initial intromission of copulatory series; $\mathrm{I}_{\mathrm{E}-1}=$ penultimate intromission of series; $\mathrm{E}=$ ejaculation.

reflects the fact that the ICI is shortened in the absence of intromission and sexual activity typically resumed in less than $20 \mathrm{sec}$.

The forms of the rate curves following successful intromission are similar to those for mounts, but show somewhat higher initial values and peaks, a gradual decline, and an intermediate resting level: the ICI usually outlasts the $20-$ sec. recording period. The penultimate post-intromission recordings $\left(\mathrm{I}_{\mathrm{E}-1}\right)$ in the sequence maintain a slightly higher level throughout than those obtained following the initial intromission $\left(\mathrm{I}_{1}\right)$, though the difference is not statistically significant. The curve describing heart rate following intromission with ejaculation (E) is distinct from the other three, showing a sustained high rate which does not begin to decline until 14 sec. after coitus and does not reach an asymptote at all within the 20-sec. recording period.

In some respects, the female heart rate data resemble the male's. The post-mount rate increase is small and transient; the functions for intromissions are alike, have higher peak rates, and are sustained longer. The postejaculatory rate has the highest rate and is sustained longest. Unlike the male, the peak rate for $I_{1}$ is higher than for $\mathrm{I}_{\mathrm{E}-1}$, but the latter maintains a level well above that of $\mathrm{I}_{1}$ throughout the $20-\mathrm{sec}$. period.

It is interesting that the recovery of rate after abortive mounts appears some $3 \mathrm{sec}$. earlier in the female data than in the male's. This is a reflection of the fact that it is often the female who initiates sexual activity following mounts without intromission, and that when she does, it happens earlier in the ICI than when initiated by the male.

Analysis of variance (Winer, 1962) was performed on the data in order to determine if there was a significant difference between the heart rate measured after the first intromission in the series, $\mathrm{I}_{1}$, and the last, $\mathrm{I}_{\mathrm{E}-1}$. The measure of heart rate used was the level at $10 \mathrm{sec}$. following the termination of the coital contact. This 10-sec. point falls roughly in the middle of the ICI. It was chosen for its freedom from transient rate effects caused by coital effort and movement, and from activity initiating the next mount. The statistical analysis did not show any significant differences between $I_{1}$ and $I_{E-1}$ for either the males or the females.

Further statistical analysis was applied to determine whether heart rate discriminated the major sexual events in the sequence (M, I, and E) from each other. The Duncan Range Test for multiple comparisons was used to insure the proper alpha level (Winer, 1962). For the males, heart rate $10 \mathrm{sec}$. following ejaculation was significantly higher $(\mathrm{P} \leq .05)$ than after $\mathrm{M}$ or I. For the females, the curve following mounts without intromission was significantly lower $(P \leq .05)$ than after I or $\mathrm{E}$.

\section{Diseussion}

Although the trend was in the proper direction, the failure of early and late intromissions to affect distinctively the postcopulatory rate recommends a reconsideration of the hypothesis of successive increments in sexual excitatory states with successive intromissions. Alternatively, changes in heart rate may not be a sufficiently sensitive index of these states. However, the results of this experiment corroborate others in demonstrating clear relationships of peak rate and pattern to observable sexual events and presumably to central excitory states accompanying these.

\section{References}

BEACH, F. A., \& WHALEN, R. E. Effects of intromission without ejaculation upon sexual behavior in male rats. J. comp. physiol. Psychol., 1959, 52, 476-481.

BOAS, E., \& GOLDSCHMIDT. E. The heart rate. Springfield: C. C. Thomas, 1932.

FORD, C. S., \& BEACH, F. Patterns of sexual behavior. London: Eyre \& Spottiswood, 1952.

LARSSON, K. Conditioning and sexual behavior in the male albino rat. Stockholm: Almqvist \& Wiksell, 1956.

MENDELSOHN, M. Deutsche Medizenische Wochenschrift, 1896, 22, 383.

WINER, B. Statistical principles in experimental design. San Francisco: McGraw-Hill, 1962.

\section{Note}

1. This research was supported by grant No. MH-5207 from NIMH and CHHD to Dr. O. Thomas Law. Early exploratory work was supported by the NAS-NRC Committee on Research in Problems of Sex. 